Galián Nicolás, B., García Sanz, M.P. \& Belmonte Almagro, M.L. (2018). Evaluación de la participación familiar en el proceso educativo de los discentes desde la percepción del profesorado. Revista Electrónica Interuniversitaria de Formación del Profesorado, 21(3), 45-62.

\title{
Evaluación de la participación familiar en el proceso educativo de los discentes desde la percepción del profesorado
}

\author{
Begoña Galián Nicolás, María Paz García Sanz y María Luisa Belmonte Almagro \\ Universidad de Murcia
}

\section{Resumen}

Actualmente se considera un factor de calidad educativa la existencia de una buena relación entre la familia y la escuela, siendo los docentes quienes realizan el papel de mediadores entre los progenitores del alumnado y el centro. Por ello, con el presente trabajo, mediante una investigación descriptiva de carácter evaluativo, a través de un cuestionario, se ha querido conocer la percepción que tiene el profesorado sobre la participación de las familias en la educación de los hijos. Tras un muestreo aleatorio estratificado se ha contado con la participación de 758 docentes de 271 centros de Educación Infantil, Primaria y Secundaria. Los resultados ponen de manifiesto que, de forma general los docentes consideran que la participación es moderada a nivel no normativo y baja a nivel normativo, que existen diferencias significativas en función de las variables consideradas en la investigación y que se han obtenido dos perfiles de profesores según su consideración de la participación de las familias en la educación de los discentes.

\section{Palabras clave}

Participación de los padres; escuela; perfil docente; familia.

\section{Contacto:}

Begoña Galián Nicolás, begona.g.n@um.es. 


\title{
Evaluation of family participation in the educational process of students from the teachers' perspective
}

\begin{abstract}
Nowadays, the existence of a good relationship between the family and the school is considered a factor of educational quality. The teachers are the ones who perform the role of mediators between the parents of the students and the centre. Therefore, the aim of this descriptive and evaluative research is to get to know, by means of a questionnaire, the teachers' perception on the involvement of parents in the educational process of their children. The participation of 758 teachers of 271 pre-school, primary and secondary educational centres has been necessary in order to carry out a stratified random sample. The results show that, in general, teachers consider that there is a moderated participation at a non-normative level and a low participation at a normative one, as well as the existence of significant differences in accordance with the variables listed in the present research and the collection of two teacher profiles depending on their consideration regarding families' involvement in the educational process of their children.
\end{abstract}

\section{Key words}

Parents' involvement; school; teacher profile; family.

\section{Introducción}

"La escuela participativa está concebida como una comunidad integrada por padres, profesores, alumnos y personal no docente" (Gómez Llorente, 2006, p.18), pero la educación de los niños va mucho más allá. No existe un solo contexto de educación, sino que en él interfieren escuela, profesorado, familia, medios de comunicación, entorno, etc. (Bolivar, 2006). Es de gran importancia que todos estos agentes trabajen de forma coherente y cohesionada para que se produzcan en el alumnado los resultados de aprendizaje necesarios (López Barrero, 2010; Sheldon y Epstein, 2005; Vila, 1998). En especial la familia y la escuela (Solé, 1996), dado que el trabajo coordinado entre ambos ayuda a producir efectos positivos en el educando (Gomariz, Parra, García-Sanz, HernándezPrados y Pérez Cobacho, 2008), y por ello debe primar la corresponsabilidad compartida entre los padres y el resto de la comunidad educativa, ejerciendo ambos una participación directa y responsable (Bolivar, 2006) y creando una visión colectiva de la educación (Eisner, 2002).

"La familia es la institución primaria en la que el ser humano es criado, y ocupa, junto a la escuela, un lugar fundamental en lo que a su educación y formación se refiere" (López Barrero, 2010, p.154). El modelo de organización familiar se ha ido modificando con el paso de los años (Bas y Pérez Guzmán, 2010). La idea de la familia, concebida desde una perspectiva tradicional, ya no representa la realidad de España (García Olalla, 2003). El énfasis está en que la familia, sea cual sea su forma, ha de ser el lugar donde crezcan las nuevas generaciones adecuadamente y así poder afrontar las dificultades que se presentan a lo largo de la vida. Ello implica la creación de un espacio de convivencia agradable donde 
exista un sentimiento de pertenencia y acogimiento (Mínguez, 2014). En definitiva, actualmente no podemos hablar de un concepto de familia generalizable, empezando por hablar de familias en plural (Gomariz et al., 2008).

Se sabe que el niño se relaciona sobre todo en la familia y en la escuela (Solé, 1996) y que sendas instituciones se complementan con el objetivo de lograr la educación del menor (Mérida, 2002). Por ello se hace evidente la necesidad de que exista una buena relación entre ambos contextos (Llevot y Bernad, 2015), incluyendo una participación efectiva. Según De la Guardia y Kñallinsky (2007), para conseguir una educación de calidad es imprescindible la participación de las familias. Esta participación se refiere a las actividades, individuales o grupales, que se realizan con la finalidad de resolver un problema y lograr una comunidad más democrática (Llevot y Bernad, 2015). A nivel educativo, se considera como la intervención activa en las decisiones y actuaciones relativas a todo el proceso educativo que se lleva a cabo, tanto en los centros como en el aula (García Albaladejo y Sánchez Liarte, 2006; Santos Guerra, 1999), siendo esta un indicador de calidad (Martínez González et al., 2002).

Con respecto al cómo participar, no existe un modelo de participación educativa que se considere universal, pero entre los más destacados se encuentra el modelo de Epstein et al. (2002) que defiende que la calidad de la educación depende de las interacciones que se crean entre la escuela y el resto de implicados, planteando una consideración holística de la participación, y clasificándola en niveles (parental, comunicativa, voluntariado, enseñanza en casa y colaboración con la comunidad). Otro modelo destacado es el de Vogels (2002), que parte de cuatro perfiles familiares según la relación que se establece con el centro (consumidores, clientes, participantes y socios). En base a estos dos modelos, García-Sanz, Hernández-Prados, Parra y Gomariz (2016), encontraron cuatro perfiles de participación en Educación Primaria y Parra, Gomariz, Hernández-Prados y García-Sanz (2017), otros cuatro en Educación Infantil.

En la actualidad la participación de las familias en los centros educativos además de un deber moral es un derecho, y como tal, los poderes públicos han de trabajar para lograr que ese derecho se cumpla (Repáraz y Naval, 2014). La Ley Orgánica 8/2013, de 9 de diciembre, para la Mejora de la Calidad Educativa (LOMCE, 2013) considera a los padres los primeros responsables de la educación de sus hijos, y regula los aspectos de la participación normativa, que se organizan en las Asociaciones de Padres y Madres de Alumnos y los Consejos Escolares de Centros.

Los docentes entienden que la participación de las familias es un factor clave para que se produzca el éxito de los alumnos (Andrés y Giró, 2016; Llevot y Bernad, 2015), aunque a veces el profesorado marque las distancias con las familias debido a que puede considerar que la intervención de los padres dificulta su labor docente (Consejo Escolar Del Estado, 2015).

El profesorado actúa como intermediario entre la escuela y la familia, y su percepción sobre la participación de los padres puede condicionar la enseñanza (Gomila y Pascual, 2015). De acuerdo con Mehlig y Shumow (2013) y Miller, Lines, Sullivan y Hermanutz (2013), el profesorado debe estar suficientemente formado para poder facilitar una participación significativa de las familias, más aun teniendo en cuenta que los maestros siguen afirmando que uno de los retos más importantes encontrados al incorporarse a la vida laboral es el de establecer relaciones de calidad (Evans, 2013).

A partir de una investigación más amplia coordinada por el Consejo Escolar del Estado (2014), el objetivo general del que se partió en este estudio fue analizar la percepción que 
tienen los docentes sobre la participación de las familias en la educación de hijos. Para ello se plantearon los siguientes objetivos específicos:

1. Describir el nivel de participación de las familias en el proceso educativo de sus hijos desde la perspectiva del profesorado.

2. Analizar la posible existencia de diferencias significativas, así como la magnitud de las mismas respecto al nivel de participación de las familias, en función de la edad del profesorado, la etapa educativa en la que éste imparte docencia y la titularidad del centro.

3. Obtener perfiles docentes, según la percepción que éstos poseen sobre la participación de las familias en la educación de los hijos, considerando variables relevantes en la investigación.

\section{Metodología}

\section{Participantes}

La selección de los participantes se llevó a cabo mediante un muestreo aleatorio estratificado en función de la comunidad autónoma, la etapa educativa y la titularidad del centro. En esta investigación se ha obtenido respuesta de 758 docentes, pertenecientes a 271 centros de Educación Infantil, Primaria y Secundaria.

\section{Instrumento de recogida de información y variables}

En la investigación más amplia a la que nos hemos referido, se utilizó un cuestionario dirigido al profesorado integrado por 70 ítems de diferentes tipos. Para este estudio se han seleccionado 11 ítems de participación familiar que hacen referencia a la percepción que tienen los docentes sobre la participación directa de las familias desde el hogar, así como su implicación en la AMPA y en el Consejo Escolar del centro (CEC). Se trata de ítems cerrados con una escala numérica de 4 grados (1: nunca; 2: algunas veces; 3: frecuentemente; 4: siempre), cuyo contenido es el siguiente:

Las familias:

P1: Apoyan y supervisan las tareas escolares de sus hijos.

P2: Se interesan por la asistencia de sus hijos al centro.

P3: Animan a sus hijos a participar en las actividades organizadas por el centro.

P4: Fomentan la realización de actividades culturales y formativas.

P5: Perciben la educación de sus hijos como un valor fundamental.

P6: Promueven la autonomía y responsabilidad de sus hijos en el estudio.

P7: Fomentan el buen clima de estudio desde el hogar.

P8: Me apoyan en la función docente.

Po: Facilitan la interacción educativa de sus hijos.

P10: Participan en las actividades organizadas por la AMPA.

P11: Participan en las elecciones de representante de las familias en el CEC. 
La media de estos ítems conforma la variable criterio de la investigación, denominada participación familiar en la educación de los hijos.

También se han utilizado 3 ítems de carácter sociodemográfico que constituyen las variables predictoras del estudio. Dichas variables hacen referencia a la edad del docente, titularidad del centro y etapa educativa.

El cuestionario completo fue elaborado por expertos y validado por profesores de cinco universidades españolas. Se calculó la validez de constructo de la parte del cuestionario utilizada en esta investigación mediante un análisis factorial exploratorio de componentes principales. Una vez comprobada la adecuación de la muestra (Medida $\mathrm{KMO}=.902$ y Prueba de esfericidad de Bartlett, $\mathrm{P}=.000$ ), con el $60,524 \%$ de varianza explicada, se obtuvieron dos factores (Tabla 1).

Tabla 1.

Factores obtenidos mediante análisis factorial

\begin{tabular}{|c|c|c|c|c|c|c|c|c|c|c|c|}
\hline \multirow{2}{*}{ Componentes } & \multicolumn{10}{|c|}{ Ítems } \\
& P1 & P2 & P3 & P4 & P5 & P6 & P7 & P8 & P9 & P10 & P11 \\
\hline 1 &, 824 &, 792 &, 781 &, 749 &, 747 &, 724 &, 722 &, 706 &, 617 &, 093 &, 192 \\
\hline 2 &, 076 &, 036 &, 113 &, 083 &, 275 &, 105 &, 192 &, 245 &, 183 &, 856 &, 822 \\
\hline
\end{tabular}

Como se aprecia, el factor 1 integra ítems relacionados con una participación educativa no normativa (ítems del 1 al 9), mientras que el factor 2 queda conformado por los dos ítems que se refieren a la participación normativa, es decir, participación de las familias en la AMPA del centro y en el CEC.

Respecto a la fiabilidad de los ítems utilizados en este trabajo se analizó mediante el coeficiente alpha de Cronbach, con un resultado de 0,887 , por lo que se puede decir que el nivel de fiabilidad es muy satisfactorio (DeVellis, 2003).

\section{Método, procedimiento y Técnicas de análisis de datos}

La investigación supone un estudio cuantitativo-descriptivo-no experimental, de carácter exploratorio y evaluativo, ya que, de acuerdo con el ciclo de intervención educativa (GarcíaSanz, 2012), sus resultados podrán ser utilizados como una evaluación de necesidades para diseñar programas de intervención.

En relación al procedimiento, el Consejo Escolar del Estado solicitó el desarrollo de un estudio en general sobre la familia y su participación en los centros educativos. Una vez validado el cuestionario se procedió a la administración del mismo a través de una plataforma web que se diseñó para que el profesorado participase otorgando sus respuestas. Los datos fueron analizados mediante el paquete estadístico SPSS IBM, aplicando tanto la estadística descriptiva como la inferencial paramétrica $(\alpha=, 05)$ puesto que la muestra reunía las condiciones necesarias para poder hacer uso de ella (Siegel, 1990). 


\section{Resultados}

\section{Descripción de la participación familiar en el proceso educativo de los hijos}

La Tabla 2 indica que, a nivel global, los docentes consideran que frecuentemente los padres participan en la educación de sus hijos. Con respecto a la participación no normativa (factor 1), los docentes opinan que las familias se implican frecuentemente, pero en lo referente a la participación normativa (factor 2 ), indican que sólo lo hacen algunas veces, no superando el estándar del que se ha partido $(2,5)$.

Tabla 2.

Estadísticos descriptivos globales y de cada factor

\begin{tabular}{|c|c|c|}
\hline & Media & Desviación típica \\
\hline Global & 2,83 &, 466 \\
\hline Factor 1 & 2,90 &, 497 \\
\hline Factor 2 & 2,39 &, 608 \\
\hline
\end{tabular}

De forma más concreta en la Tabla 3 se presentan las medias y desviaciones típicas referentes a cada ítem.

Tabla 3.

Medias y desviaciones típicas de los ítems del cuestionario

\begin{tabular}{|c|c|c|}
\hline Ítems & Media & Desviación típica \\
\hline $\mathrm{P} 1$ & 2,69 &, 610 \\
\hline $\mathrm{P} 2$ & 3,46 &, 600 \\
\hline $\mathrm{P} 3$ & 2,86 &, 708 \\
\hline $\mathrm{P} 4$ & 2,62 &, 740 \\
\hline $\mathrm{P} 5$ & 3,16 &, 709 \\
\hline $\mathrm{P} 6$ & 2,64 &, 660 \\
\hline $\mathrm{P} 7$ & 2,70 &, 650 \\
\hline $\mathrm{P} 8$ & 3,00 &, 658 \\
\hline $\mathrm{P} 9$ & 2,91 &, 658 \\
\hline $\mathrm{P} 10$ & 2,34 &, 677 \\
\hline $\mathrm{P} 11$ & 2,44 &, 715 \\
\hline
\end{tabular}

Como se aprecia, los ítems que destacan con promedios más altos, son, por un lado, el que hace referencia al interés de los padres por la asistencia de los hijos al centro $\left.M_{P_{2}}=3,46\right)$, valorado por los docentes con las categorías entre frecuentemente y mucho; por otro lado, el relativo a la percepción de la educación de los hijos de 
aquellos como un valor fundamental $\left(\mathrm{M}_{\mathrm{P}_{5}}=3,16\right)$, ha sido evaluado por el profesorado con la categoría de frecuentemente.

Asimismo, el hecho de que los profesores se vean apoyados en la función docente por las familias, respetando y colaborando éstas en las decisiones que se toman en el centro educativo, ha sido valorado con la categoría de frecuentemente ( $\mathrm{P}_{\mathrm{P} 8}=3,00$ ). Por el contrario se observa que los ítems que no logran llegar al umbral establecido son los referentes a la participación normativa de las familias ( $\mathrm{MP}_{10}=2,34$ y $\left.\mathrm{MP}_{11}=2,44\right)$.

Diferencias significativas y magnitud del efecto de variables predictoras.

En la Tabla 4 se indican algunos descriptivos y la significación estadística de la variable edad.

Tabla 4.

Descriptivos y resultados de la prueba ANOVA en función de la edad de los docentes

\begin{tabular}{|c|c|c|c|c|}
\hline & Grupos de edad & Media & $\begin{array}{c}\text { Desviación } \\
\text { típica }\end{array}$ & $\begin{array}{c}\text { Significación } \\
\text { estadística }\end{array}$ \\
\hline \multirow{5}{*}{ Global } & 20 y 30 años & 2,87 & , 470 & \multirow{5}{*}{,001 } \\
\hline & 31 y 40 años & 2,79 & ,447 & \\
\hline & 41 y 50 años & 2,74 & ,481 & \\
\hline & 51 y 60 años & 2,93 & ,434 & \\
\hline & Más de 60 años & 2,96 & ,612 & \\
\hline \multirow{5}{*}{ Factor 1} & 20 y 30 años & 2,93 & ,496 & \multirow{5}{*}{,001 } \\
\hline & 31 y 40 años & 2,87 & ,473 & \\
\hline & 41 y 50 años & 2,82 &, 514 & \\
\hline & 51 y 60 años & 3,02 & , 476 & \\
\hline & Más de 60 años & 3,03 & ,665 & \\
\hline \multirow{5}{*}{ Factor 2} & 20 y 30 años & 2,55 & ,652 & \multirow{5}{*}{,035 } \\
\hline & 31 y 40 años & 2,38 & ,634 & \\
\hline & 41 y 50 años & 2,30 &, 587 & \\
\hline & 51 y 60 años & 2,43 &, 534 & \\
\hline & Más de 60 años & 2,56 &, 729 & \\
\hline
\end{tabular}

El hecho de haber encontrado diferencias estadísticamente significativas, tanto a nivel global como por factores, sólo indica que es poco probable que dichas diferencias sean debidas al azar y por tanto, las mismas pueden ser aceptadas como reales. Sin embargo, la significación estadística no proporciona información sobre la magnitud o fuerza con la que se producen dichas diferencias. Por ello, se ha procedido a calcular el estadístico "d" de Cohen como medida del denominado tamaño o magnitud del efecto (Cohen, 1988). La interpretación de dicha medida es la que se muestra en la Tabla 5. 
Tabla 5 .

Interpretación del estadístico "d" de Cohen (1988)

\begin{tabular}{|l|c|}
\hline \multicolumn{1}{|c|}{ Valoración } & Índice $\boldsymbol{d}$ \\
\hline Efecto nulo & 0 \\
\hline Efecto bajo & $\pm 0,20$ \\
\hline Efecto medio & $\pm 0,50$ \\
\hline Efecto alto & $\pm 0,80$ \\
\hline
\end{tabular}

En relación a la edad, la Tabla 6 muestra que, ni a nivel global ni por factores, en ninguno de los pares de grupos comparados en los que se han encontrado diferencias significativas, se ha obtenido un tamaño del efecto considerado como típico o medio.

Tabla 6.

Resultados de la " $d$ " de Cohen en relación a la edad de los docentes

\begin{tabular}{|c|c|c|}
\hline \multirow{2}{*}{ Global } & $\begin{array}{c}\text { Grupos con diferencias } \\
\text { significativas }\end{array}$ & d de Cohen \\
\hline \multirow{2}{*}{ Factor 1} & Entre $31-40$ y $51-60$ años & $\pm 0,3073$ \\
\cline { 2 - 3 } & Entre $41-50$ y $51-60$ años & \pm 0.4245 \\
\cline { 2 - 3 } & Entre $31-40$ y $51-60$ años & \pm 0.3075 \\
\hline \multirow{2}{*}{ Factor 2} & Entre $20-50$ y $51-60$ añ $41-50$ años & \pm 0.4009 \\
\hline
\end{tabular}

En la Tabla 7 se presentan los descriptivos y significación estadística de la variable etapa educativa.

Tabla 7.

Descriptivos y resultados de la prueba ANOVA en función de la etapa educativa

\begin{tabular}{|c|c|c|c|c|}
\hline & $\begin{array}{c}\text { Etapa } \\
\text { educativa }\end{array}$ & Media & $\begin{array}{c}\text { Desviación } \\
\text { típica }\end{array}$ & $\begin{array}{c}\text { Significación } \\
\text { estadística }\end{array}$ \\
\hline \multirow{3}{*}{ Global } & Infantil & 2,92 &, 519 & \multirow{3}{*}{,000 } \\
\hline & Primaria & 2,92 &, 435 & \\
\hline & Secundaria & 2,63 & ,412 & \\
\hline \multirow{3}{*}{ Factor 1} & Infantil & 3,01 &, 563 & \multirow{3}{*}{,000 } \\
\hline & Primaria & 2,99 &, 462 & \\
\hline & Secundaria & 2,71 & ,444 & \\
\hline \multirow{3}{*}{ Factor 2} & Infantil & 2,45 &, 685 & \multirow{3}{*}{,000 } \\
\hline & Primaria & 2,49 &, 605 & \\
\hline & Secundaria & 2,20 &, 509 & \\
\hline
\end{tabular}


También en esta ocasión se aprecian diferencias significativas de participación en función de la etapa educativa en la que el profesorado imparte docencia. En la Tabla 8 se observa que, tanto a nivel global como en el factor 1 , se supera el tamaño del efecto considerado como típico o medio entre los grupos con significación estadística. Sin embargo, en el factor 2 no se ha alcanzado dicho valor al comparar las etapas de Infantil y Secundaria.

Tabla 8.

Resultados de la " $d$ " de Cohen en relación a la etapa educativa

\begin{tabular}{|c|c|c|}
\hline & $\begin{array}{c}\text { Grupos con diferencias } \\
\text { significativas }\end{array}$ & $\begin{array}{c}d \text { de } \\
\text { Cohen }\end{array}$ \\
\hline \multirow{3}{*}{ Global } & Infantil y Secundaria & $\pm 0,6296$ \\
\cline { 2 - 3 } & Primaria y Secundaria & $\pm 0,6822$ \\
\hline \multirow{2}{*}{ Factor 1 } & Infantil y Secundaria & $\pm 0,5845$ \\
\cline { 2 - 3 } & Primaria y Secundaria & $\pm 0,6082$ \\
\hline \multirow{2}{*}{ Factor 2 } & Infantil y Secundaria & $\pm 0,4110$ \\
\cline { 2 - 3 } & Primaria y Secundaria & $\pm 0,5151$ \\
\hline
\end{tabular}

En cuanto a las diferencias encontradas en función de la titularidad del centro de los docentes, en la Tabla 9 se muestran algunos descriptivos y la significación estadística.

Tabla 9.

Descriptivos y resultados de la prueba ANOVA en función de la titularidad del centro

\begin{tabular}{|c|c|c|c|c|}
\hline & $\begin{array}{c}\text { Etapa } \\
\text { educativa }\end{array}$ & Media & $\begin{array}{c}\text { Desviación } \\
\text { típica }\end{array}$ & $\begin{array}{c}\text { Significación } \\
\text { estadística }\end{array}$ \\
\hline \multirow{3}{*}{ Global } & Público & 2,824 & , 487 & \multirow{3}{*}{,030 } \\
\hline & concertado & 2,811 & ,423 & \\
\hline & Privado & 3,072 & ,497 & \\
\hline \multirow{3}{*}{ Factor 1} & Público & 2,892 &, 513 & \multirow{3}{*}{,008 } \\
\hline & concertado & 2,897 & ,459 & \\
\hline & Privado & 3,213 &, 531 & \\
\hline \multirow{3}{*}{ Factor 2} & Público & 2,424 & ,629 & \multirow{3}{*}{,180 } \\
\hline & concertado & 2,347 &, 554 & \\
\hline & Privado & 2,283 & ,736 & \\
\hline
\end{tabular}

Como se aprecia, tanto a nivel global como en el factor 1, existen diferencias significativas en función del tipo de centro, en cambio, en el factor 2 no se aprecia significación estadística entre ninguno de los diferentes tipos de centros. 
Por último, respecto al cálculo de la "d" de Cohen para averiguar la magnitud de las diferencias significativas halladas, en la Tabla 10 se muestra que dicho índice supera el tamaño del efecto considerado como medio, tanto a nivel global como en el factor 1.

Tabla 10.

Resultados de la " $d$ " de Cohen en relación a la titularidad del centro

\begin{tabular}{|c|c|c|}
\hline \multirow{3}{*}{ Global } & $\begin{array}{c}\text { Grupos con diferencias } \\
\text { significativas }\end{array}$ & $d$ de Cohen \\
\cline { 2 - 3 } & Privada y Pública & $\pm 0,5040$ \\
\hline \multirow{3}{*}{ Factor 1} & Privada y Concertada & $\pm 0,5656$ \\
\cline { 2 - 3 } & Privada y Pública & $\pm 0,6149$ \\
\hline
\end{tabular}

\section{Perfiles de percepción docente sobre participación familiar}

Para responder al tercer y último objetivo de la investigación, consistente en obtener los perfiles docentes en relación a la participación de las familias en la educación de los hijos, se han considerado las variables categóricas: edad del docente, etapa educativa y titularidad del centro, así como las variables numéricas: participación familiar no normativa y participación familiar normativa.

Tras realizar el correspondiente análisis cluster bietápico, en la Tabla 11 se observa que se han obtenido dos perfiles docentes. En dicha tabla se aprecia que de los 758 participantes, se han producido 45 casos no válidos, por lo que la configuración de los conglomerados se ha generado con 713 docentes.

Tabla 11

Distribución de perfiles docentes

\begin{tabular}{|c|c|c|c|c|}
\hline \multicolumn{2}{|c|}{} & N & $\begin{array}{c}\text { \% de } \\
\text { combinados }\end{array}$ & $\begin{array}{c}\text { \% del } \\
\text { total }\end{array}$ \\
\hline \multirow{3}{*}{ Perfil } & 1 & 393 & $55,1 \%$ & $51,8 \%$ \\
\cline { 2 - 5 } & 2 & 320 & $44,9 \%$ & $42,2 \%$ \\
\cline { 2 - 5 } & Combinados & 713 & $100,0 \%$ & $94,1 \%$ \\
\hline \multicolumn{2}{|c|}{ Casos excluidos } & 45 & & $5,9 \%$ \\
\hline \multicolumn{2}{|c|}{ Total } & 758 & & $100,0 \%$ \\
\hline \multicolumn{2}{|c|}{} \\
\hline \multicolumn{2}{|c|}{}
\end{tabular}

Seguidamente se muestran una serie de tablas que servirán para la interpretación de los perfiles docentes conformados. 
Tabla 12.

Media y desviación típica de las variables numéricas por perfiles

\begin{tabular}{|c|c|c|c|c|c|}
\hline \multicolumn{2}{|c|}{} & \multicolumn{2}{c|}{ Participación no normativa } & \multicolumn{2}{c|}{ Participación normativa } \\
\cline { 2 - 6 } \multicolumn{2}{|c|}{} & Media & Desv. típica & Media & Desv. típica \\
\hline \multirow{3}{*}{ Perfil } & 1 & 2,80 &, 522 & 2,28 &, 578 \\
\cline { 2 - 6 } & 2 & 2,99 &, 456 & 2,52 &, 607 \\
\cline { 2 - 6 } & Combinados & 2,89 &, 501 & 2,39 &, 603 \\
\hline
\end{tabular}

Tabla 13.

Frecuencias y porcentajes según edad del profesorado por perfiles

\begin{tabular}{|c|c|c|c|c|c|c|c|c|c|c|c|}
\hline \multicolumn{12}{|c|}{ Edad del profesorado } \\
\hline & & \multicolumn{2}{|c|}{20 -30 años } & \multicolumn{2}{|c|}{ 31-40 años } & \multicolumn{2}{|c|}{ 41-50 años } & \multicolumn{2}{|c|}{ 51-60 años } & \multicolumn{2}{|c|}{ Más de 60 años } \\
\hline & & $\mathbf{F}$ & $\%$ & $\mathbf{F}$ & $\%$ & $\mathbf{F}$ & $\%$ & $\mathbf{F}$ & $\%$ & $\mathbf{F}$ & $\%$ \\
\hline \multirow{3}{*}{ Perfil } & 1 & 26 & 41,3 & 163 & 57,8 & 123 & 66,8 & 74 & 41,8 & 7 & 100,0 \\
\hline & 2 & 37 & 58,7 & 119 & 42,2 & 61 & 33,2 & 103 & 58,2 & 0 &, 0 \\
\hline & Comb. & 63 & 100,0 & 282 & 100,0 & 184 & 100,0 & 177 & 100,0 & 7 & 100,0 \\
\hline
\end{tabular}

Tabla 14.

Frecuencias y porcentajes según etapa educativa por perfiles

\begin{tabular}{|c|c|c|c|c|c|c|c|}
\hline \multicolumn{2}{|c|}{} & \multicolumn{2}{c|}{ Infantil } & \multicolumn{2}{c|}{ Primaria } & \multicolumn{2}{c|}{ Secundaria } \\
\cline { 2 - 8 } & & F & $\%$ & F & $\%$ & F & $\%$ \\
\hline \multirow{3}{*}{} & 1 & 146 & 99,3 & 20 & 5,9 & 227 & 100,0 \\
\cline { 2 - 8 } & 2 & 1 &, 7 & 319 & 94,1 & 0 &, 0 \\
\cline { 2 - 8 } & Combinados & 147 & 100,0 & 339 & 100,0 & 227 & 100,0 \\
\hline
\end{tabular}

Tabla 15.

Frecuencias y porcentajes según la titularidad del centro por perfiles

\begin{tabular}{|c|c|c|c|c|c|c|c|}
\hline \multicolumn{2}{|c|}{} & \multicolumn{2}{c|}{ Pública } & \multicolumn{2}{c|}{ Concertada } & \multicolumn{2}{c|}{ Privada } \\
\cline { 2 - 8 } & & F & $\%$ & F & $\%$ & F & $\%$ \\
\hline \multirow{2}{*}{ Perfil } & 1 & 235 & 55,6 & 135 & 50,6 & 23 & 100,0 \\
\cline { 2 - 8 } & 2 & 188 & 44,4 & 132 & 49,4 & 0 &, 0 \\
\hline & Combinados & 423 & 100,0 & 267 & 100,0 & 23 & 100,0 \\
\hline
\end{tabular}




\section{Características del perfil docente 1}

El primer perfil integra el $55,1 \%$ de los docentes (Tabla 11). Está constituido fundamentalmente por profesorado que tiene entre 31-40 años, 41-50 años y más de 60 años (Tabla 13). Se trata de profesores que imparten docencia fundamentalmente en las etapas de Infantil y Secundaria (Tabla 14), pertenecientes a centros de titularidad pública, concertada y privada (Tabla 15).

Los docentes que conforman el perfil 1 perciben que las familias realizan una participación no normativa próxima a alta $(M=2,80)$, es decir, colaboran en un nivel considerado casi frecuente en la educación de sus hijos de manera directa, principalmente desde el hogar. Sin embargo, se observa que dichos docentes aprecian que la implicación de las familias en la AMPA y en el CEC (participación normativa) es escasa $(M=2,28)$, es decir este tipo de participación la practican solo algunas veces (Tabla 12).

Como muestra la Figura 1, las variables: etapa educativa, edad de los docentes y titularidad del centro han resultado ser estadísticamente significativas para conformar este primer conglomerado (valores críticos de Chi Cuadrado: etapa educativa= 8,19; edad= 12,09; titularidad del centro $=8,19)$.

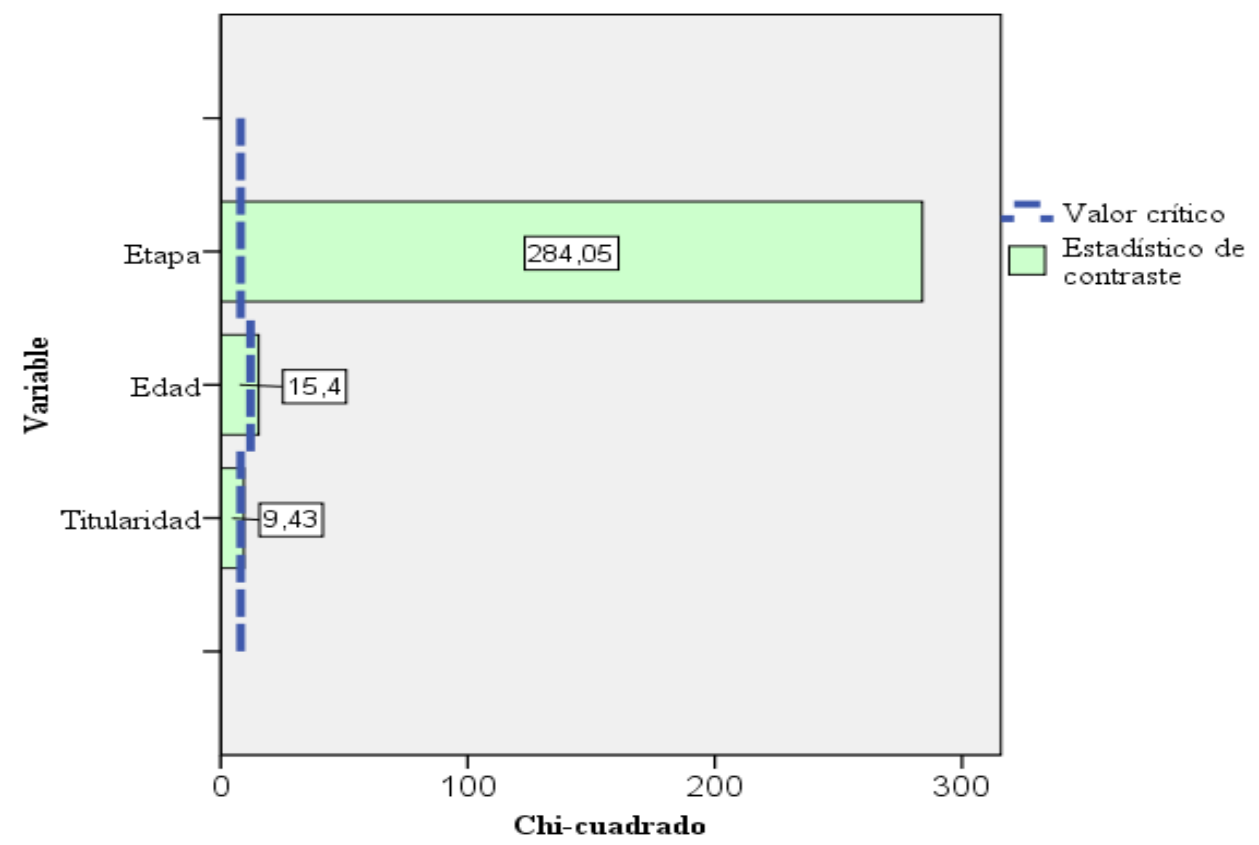

Figura 1. Importancia de las variables categóricas en el perfil docente 1

La Figura 2 muestra que las dos variables mencionadas han sido significativamente importantes para configurar este primer perfil docente, ambas con puntaciones inferiores a la media (valores críticos de T de Student de $-2,25$ y 2,25). 


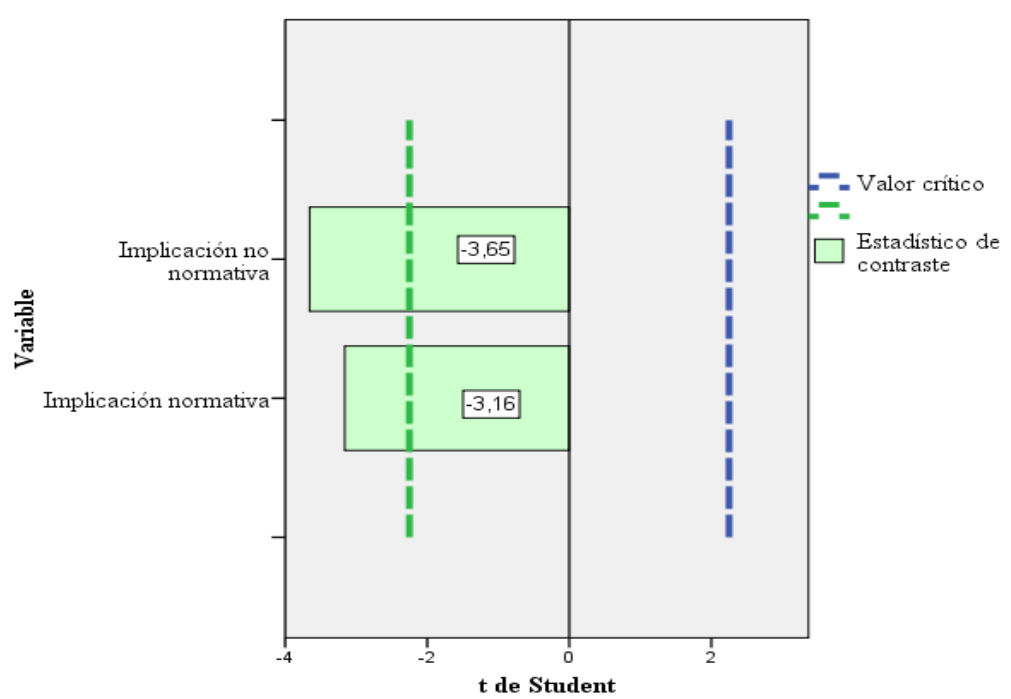

Figura 2. Importancia de las variables numéricas en el perfil docente 1

\section{Características del perfil docente 2}

El perfil 2 está compuesto por el 44,9\% de los participantes (Tabla 11). Queda conformado principalmente por docentes de entre 20-30 años y 51-60 años (Tabla 13) que imparten docencia fundamentalmente en la etapa de Primaria (Tabla 14), de centros públicos y concertados (Tabla 15).

En la Tabla 12 se aprecia que el profesorado que integra este segundo perfil de percepción docente indica que las familias del alumnado participan altamente en las tareas consideradas como no normativas $(M=2,99)$ y con una intensidad moderada en lo que respecta a su implicación en la AMPA y en el CEC $(M=2,51)$.

En la Figura 3 se observa que también en esta ocasión, han resultado ser estadísticamente significativas las variables: etapa educativa, edad de los docentes y titularidad del centro para constituir este segundo perfil (valores críticos de Chi Cuadrado: etapa educativa= 8,19; edad=12,09; titularidad del centro= 8,19).

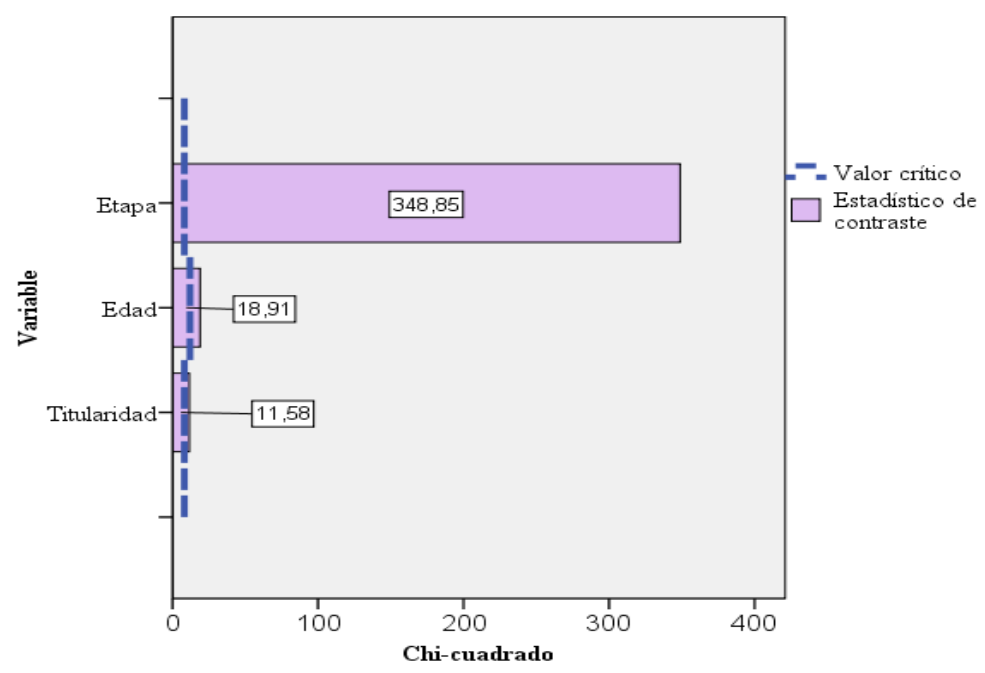

Figura 3. Importancia de las variables categóricas en el perfil docente 2 
La Figura 4 indica que, tanto la participación normativa como la no normativa han influido de forma significativa en la constitución del segundo perfil, ambas con puntuaciones superiores a la media (valores críticos de T de Student de -2,25 y 2,25).

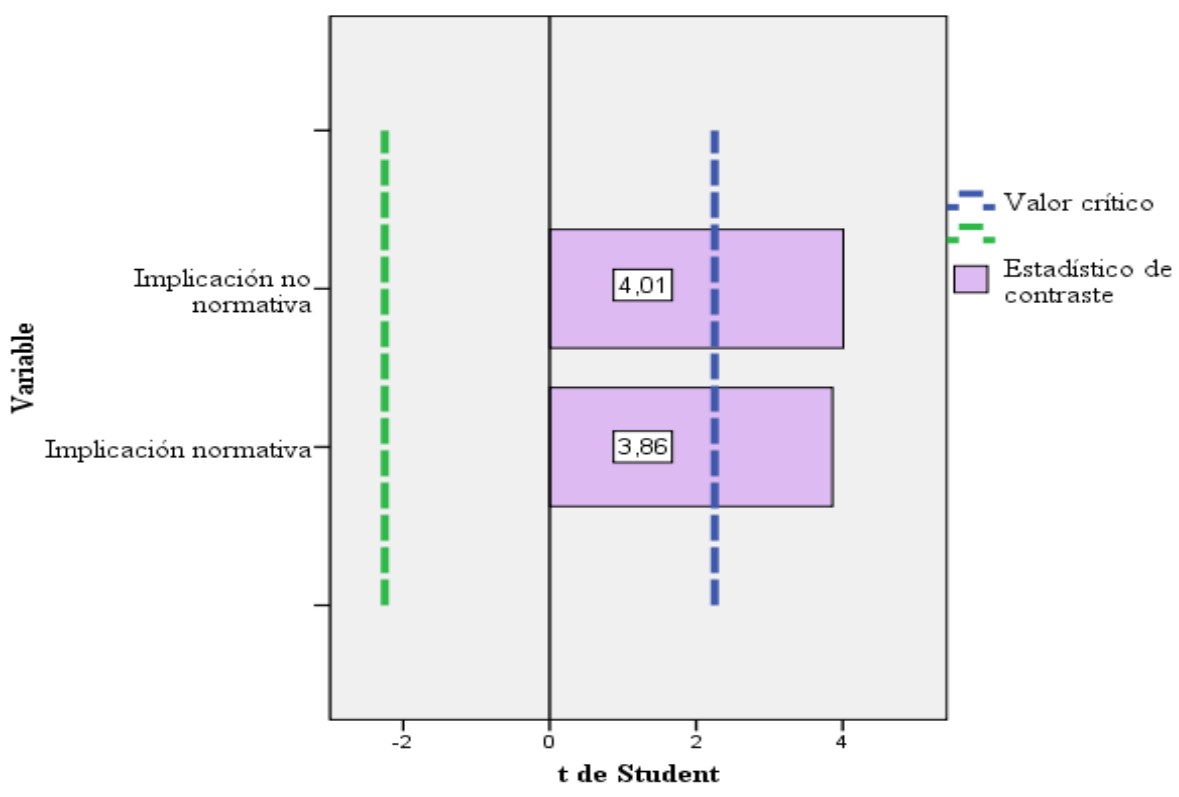

Figura 4. Importancia de las variables numéricas en el perfil docente 2

\section{Discusión y conclusiones}

Esta investigación ha permitido conocer cuál es la percepción de los docentes acerca del grado de participación de las familias en la educación de sus hijos. Epistemológicamente ha supuesto un avance, ya que se ha tenido en cuenta el punto de vista del profesorado, siendo esto poco habitual en el estudio de la relación de las familias y la escuela.

Tras analizar la percepción de los docentes acerca de la participación educativa de las familias, se ha llegado a la conclusión de que existe una participación familiar moderada en el proceso educativo de los hijos, coincidiendo con Garreta (2008).

Analizando la existencia de diferencias significativas, respecto a la participación familiar en la educación de los discentes, en las opiniones en función de la edad del profesorado se concluye que aunque no existe una relación directa que avale que a mayor edad, mayor es la consideración de la participación, sí que se percibe que existen diferencias de percepción según algunos intervalos de edad del docente. En este sentido Madrid (2005) señala que la edad del docente sí que resulta ser un factor que influye en su ejercicio profesional.

En función de la etapa educativa, el profesorado que imparte docencia en Infantil y Primaria percibe que las familias participan significativamente más en estas etapas que las que llevan a sus hijos a centros de Secundaria, según indican los docentes de esta última etapa educativa. Este resultado coincide con el obtenido por Gomariz et al., (2008), en el que se detectó un bajo nivel de participación de los padres en la vida del centro escolar, siendo menor la participación de los padres cuyos hijos cursan Educación Secundaria Obligatoria y Bachillerato, que aquellos cuyos hijos están matriculados en Educación Infantil y Primaria.

Asimismo, existen diferencias significativas según la titularidad del centro. Concretamente, los docentes que imparten docencia en centros privados consideran que los padres se 
implican mucho más en la educación no normativa, que las familias que llevan a sus hijos a centros públicos o concertados. En cambio, con respecto a la participación normativa, no se ha encontrado significación estadística entre los tres tipos de centros. Por ello, según la opinión docente, en los centros privados los padres se interesan más por la educación de sus hijos, pero participan de forma similar que en otros centros en actividades de AMPA y CEC. Todo ello guarda relación con el estudio de Jeynes (2011), en el que se expone que el tipo de centro y de participación de los padres tiene efectos en el rendimiento de los alumnos.

Finalmente, en relación a la participación de las familias en la educación de los hijos, se han encontrado dos perfiles de percepción docente. El primer perfil se compone, en su mayoría, de profesores de entre 31 y 50 años y de más de 60. Las etapas educativas en las que imparten docencia son las de Educación Infantil y Secundaria y pertenecen a los tres tipos de titularidad de centros que tenemos en España. Destaca que en este perfil se encuentran todos los docentes de los centros privados. De forma global perciben una menor participación de los padres que los pertenecientes al segundo perfil. Este por su parte considera que los padres participan más, tanto a nivel normativo como no normativo. Está compuesto por los profesores más jóvenes que tienen entre 20 y 30 años y por los que tienen entre 51 y 60. Además estos docentes imparten docencia en la etapa de Primaria y únicamente en los centros públicos y concertados. En ambos casos si por parte del centro se potencian las relaciones con las familias se obtendrán beneficios para los estudiantes a nivel global (Cox, 2005).

A modo de recapitulación, concluir que el descubrimiento de estos perfiles de percepción de participación familiar presenta el comienzo de otras líneas de investigación dirigidas a detectar la influencia de estos perfiles en distintos aspectos de la educación y en definitiva en el aprendizaje integral del alumno. Se podrá comprobar si existe relación entre los perfiles y la obtención de resultados de los alumnos, el sentimiento de pertenencia al centro, la metodología docente, la relación que mantienen con los padres, la participación de diferentes tipos de familias, etc.

\section{Referencias}

Andrés, S. y Giró, J. (2016). El papel y la representación del profesorado en la participación de las familias en la escuela. Revista electrónica interuniversitaria de formación del profesorado, 19(1), 61-71. Doi: http://dx.doi.org/10.6018/reifop.19.1.245461

Bas, E. y Pérez Guzmán, M.V. (2010). Desafíos de la familia actual ante la escuela y las tecnologías de información y comunicación. Educatio Siglo XXI, 28(1), 41-68. Recuperado de http://revistas.um.es/educatio/article/view/109721/104411

Bolivar, A. (2006). Familia y escuela: dos mundos llamados a trabajar en común. Revista de Educación 339, 119-146. Recuperado de https://dialnet.unirioja.es/servlet/articulo?codigo $=2057208$

Cohen J. (1988). Statistical Power Analysis for the Behavioral Sciences. ( $2^{\mathrm{a}}$ ed). New York: Academic Press

Consejo Escolar Del Estado (2015). La participación de las familias en la educación Escolar. Madrid: Ministerio de Educación, Cultura y Deporte.

Cox, D. (2005). Evidence-based interventions using homeschool collaboration. School Psychology Quarterly, 20, 473-497. 
De la Guardia Romero, R. y Kñallinsky, E. (2007). Manual docente de educación primaria. Educación familiar (sistema escolar y familia). Universidad de Las Palmas de Gran Canaria.

DeVellis, R.F. (2003). Scale development: theory and applications ( $2^{\mathrm{a}}$ ed.). Thousand Oaks, CA: Sage.

Eisner, E.W. (2002). La escuela que necesitamos. Ensayos personales. Buenos Aires: Amorrortu.

Epstein, J.L., Sanders, M.G., Simon, B.S., Salinas, K.C., Jansorn, N. R., y Van Voorhis, F.L. (2002). School, family, and community partnerships: Your handbook for action $\left(2^{\mathrm{a}}\right.$ ed.). Thousand Oaks, CA: Corwin.

Evans, M.P. (2013). Educating preservice teachers for family, school, and community engagement. Teaching Education, 24(2), 123-133. Doi: http://dx.doi.org/10.1080/10476210.2013.786897

García Albaladejo, A. y Sánchez Liarte, C. (2006). La participación de los padres en los documentos institucionales de los centros educativos. En A. Sánchez Albadalejo (Coord.). Participación de las familias en la vida escolar: acciones y estrategias (pp. 149-180). Madrid: Ministerio de Educación. Recuperado de https://books.google.es/books?id=v2rUpSyWey4C\&lpg=PA149\&ots=FoSsQ3S3u\&dq=La\%20participaci\%C3\%B3n\%20de\%20los\%20padres\%20en\%20los\%20docu mentos\%20institucionales\%20de\%20los\%20centros\%20educativos\&hl=es\&pg=PA150 \#v=onepage\&q=La\%20participaci\%C3\%B3n\%20de\%20los\%20padres\%20en\%20los\%20d ocumentos $\% 2$ institucionales $\% 2$ de $\% 20$ los $\% 20$ centros $\% 20$ educativos $\& f=$ false

García Olalla, M.D. (2003). La familia como contexto de desarrollo y educación en la infancia. Letras de Deusto, 33(99), 11-28.

García Sanz, M.P. (2012). Fundamentos teóricos y metodológicos de la evaluación de programas. Murcia: DM.

García-Sanz, M.P., Hernández-Prados, M.A., Parra, J. y Gomariz, M.A. (2016). Participación familiar en la etapa de educación primaria. Perfiles Educativos, 38(154), 97-117.

Garreta, J. (2008). La participación de las familias en la escuela pública. Las Asociaciones de Madres y Padres del Alumnado. Madrid: CEAPA. Recuperado de https://www.ceapa.es/content/la-participaci\%C3\%B3n-de-las-familias-en-la-escuelap\%C3\%BAblica-0

Gomariz, M.A., Parra, J., García-Sanz, M.P., Hernández-Prados, M.A. y Pérez- Cobacho, J. (2008). La comunicación entre la familia y el centro educativo. Murcia: Consejo Escolar de la Región de Murcia. Consejería de Educación, Ciencia e Investigación.

Gómez Llorente, L. (2006). La participación. Participación educativa, 1, 18-26. Recuperado de https://sede.educacion.gob.es/publiventa/detalle.action?cod=14194

Gomila, M.A. y Pascual, B. (2015). La participación de las familias en el sistema educativo: la percepción del profesorado en formación. Revista Electrónica Interuniversitaria de Formación del Profesorado, 18(3), 99-112. Doi: http://dx.doi.org/10.6018/reifop.18.3.199321

Jeynes, W.H. (2011). Parental involvement and academic success. New York: Routledge. 
Ley Orgánica 8/2013, de 9 de diciembre, para la Mejora de la Calidad Educativa, LOMCE. (BOE, núm. 295, de 10 de diciembre de 2013). Recuperado de https://www.boe.es/boe/dias/2013/12/10/pdfs/BOE-A-2013-12886.pdf

López Barrero, R. (2010). Familia Vs. Escuela. Pedagogóa mágna, 5, 154-159. Recuperado de https://dialnet.unirioja.es/descarga/articulo/3391497.pdf.

Llevot, N. y Bernad O. (2015). La participación de las familias en la escuela: factores clave. Revista de la Asociación de Sociología de la Educación, 8(1), 57-70. Recuperado de http://www.ase.es/rase/index.php/RASE/article/view/370

Madrid, D. (2005). La edad del profesorado en los procesos de enseñanza y aprendizaje de la lengua extranjera. En J. L. Martínez Dueñas, C. Pérez Basanta, N. McLaren y L. Quereda (Eds). Towards an understanding of the English Language: Past, Present and Future. Studies in Honour of Fernando Serrano (pp. 519-530). Granada: Editorial Universidad de Granada.

Martínez Dueñas, J. L., Pérez Basanta, C., McLaren, N. y Quereda, L. (2005). Towards an understanding of the English Language: Past, Present and Future. Studies in Honour of Fernando Serrano. Granada: Editorial Universidad de Granada, pp. 519530.

Martínez González, R.A., Rodríguez, B., Peña, A., Martínez, R., García González, M.P., Donaire, B., Álvarez, A.I. y Casielles, B. (2002). Dinamización de las relaciones familia-centro escolar a través de la formación del profesorado en este campo de $\begin{array}{llll}\text { actuación. } & \text { REOP, } & 11(19), 120 . & \text { Doi: }\end{array}$ http://dx.doi.org/10.5944/reop.vol.11.num.19.2000.11326

Mehlig, L.M. y Shumow, L. (2013). How is my child doing?: Preparing pre-service teachers to engage parents through assessment. Teaching Education, 24(2), 181-194. doi: http://dx.doi.org/10.1080/10476210.2013.786892

Mérida, R. (2002) Un espacio de encuentro entre la escuela y la familia: la escuela de madres y padres, Revista de Ciencias de la Educación, 192, 441- 468.

Miller, G.E., Lines, C., Sullivan, E. y Hermanutz, K. (2013). Preparing educators to partner with families. Teaching Education, 24(2), 150-163. Doi: http://dx.doi.org/10.1080/10476210.2013.786889

Mínguez, R. (2014). Ética de la vida familiar y transmisión de valores morales. Revista de Educación, 363, 210-229. Recuperado de https://dialnet.unirioja.es/servlet/articulo?codigo $=4573036$

Parra, J., Gomariz, M.A., Hernández-Prados, M.A. y García-Sanz, M.P. (2017). La participación de las familias en educación infantil. RELIEVE, 23(1), 1-26. http://doi.org/10.7203/relieve.23.1.9258

Repáraz, C. y Naval, C. (2014). Bases conceptuales de la participación de las familias. En Consejo Escolar del Estado (Coord.), La participación de las familias en la educación escolar, (pp. 21-34). Madrid: Consejo Escolar del Estado. Ministerio de Educación, Cultura y Deporte. Recuperado de http://www.mecd.gob.es/dctm/cee/publicaciones/estudioparticipacion/estudiopar ticipacion.pdf?documentld=0901e72b81b45e35

Santos Guerra, M.A. (1999). El crisol de la participación: Estudio sobre la participación en Consejos Escolares de Centros. Málaga: Aljibe. 
Sheldon, S.B. y Epstein, J.L. (2005). Involvement counts: Family and community partnerships and mathematics achievement. The Journal of Educational Research, 98(4), 196-207. Doi: http://dx.doi.org/10.3200/JOER.98.4.196-207

Siegel, S. (1990). Estadística no paramétrica para ciencias de la conducta. México: Trillas.

Solé, I. (1996). Las relaciones entre familia y escuela. Cultura Y Educación, 8(4), 11-17. Doi: http://dx.doi.org/10.1174/11356409660561241

Vila, I. (1998). Familia y escuela: dos contextos y un solo niño. Universitat de Girona. Girona. Recuperado de https://www.researchgate.net/profile/ Vila_Ignasi/publication/264419965_Familia_y_escuela_dos_contextos_y_un_solo_ nino/links/53dd20500cf216e4210c19eo.pdf

Vogels, R. (2002). Ouders bij de Les. Betrokkenheid van Ouders bij de School van hun Kind. Den Haag: Sociaal Cultureel Planbureau. 